\title{
An Approach to Improvement of Students Learning of Computer System Engineering
}

\author{
Milos Nikolic \\ RT-RK Institute for Computer Based Systems \\ Novi Sad, Serbia \\ e-mail: milos.nikolic@rt-rk.com
}

\author{
Dusan Majstorovic, Ivan Kastelan, Nebojsa Pjevalica \\ University of Novi Sad \\ Faculty of Technical Sciences \\ Novi Sad, Serbia
}

\begin{abstract}
This paper presents an approach to the study programs applied for the subject Logic Design of Computer Systems II. Study program of this subject is based on unified embedded engineering learning platform, E2LP. E2LP intended to cover complete process of embedded systems learning, considering modular approaches for unifying education in relevant embedded system technologies. Study approach in this subject intends to be a step forward in helping students to adopt knowledge better and to have stronger understanding of the complex and multidisciplinary approach in this course. This implies splitting course in two parts, one in which the students are introduced to the environment and taught how to use it, and second in which students are fulfilling the specific task with minor assistant help.
\end{abstract}

\section{INTRODUCTION}

$\mathrm{T}$ HIS paper presents an approach to the study program for the subject Logic Design of Computer Systems II. We discuss the rationale for new approach, first results and effects of it. We are presenting also the problems that we have faced and the student opinions about this approach.

Laboratory work and practical examples are very significant in electrical engineering education. This approach is forcing students to actively participate in learning process instead of being a passive listener [1]. Embedded system engineering is becoming more wanted profession since the computer based embedded systems have more important role in computer based embedded systems [2]. For these and many other reasons many technical faculties have put more emphasis on embedded systems learning by introducing a number of active learning laboratory-based courses [1].

Efficiency of laboratory work in embedded systems learning usually suffers from introduced overhead in both time and effort needed to get students familiar with hardware platforms and software tools for each course. Because of that, it is sometimes quite difficult to steer the student's attention from tools to fundamental principles.

Based on these observations, and building on comprehensive industrial experience in platform-based

The research leading to these results has received funding from the European Union's Seventh Framework Programme (FP7/2007-2013) under grant agreement $\mathrm{n}^{\circ} 317882$. engineering, a modular common design platform has been developed. The aim of the platform is to increase efficiency of laboratory-based courses. The platform is designed to cover all aspects of embedded systems learning: (1) Digital system design, (2) Computer system design, (3) Digital signal processing of audio, video and data signal streams in real-time, (4) Computer networks and interfaces and (5) System integration.

Approach based on laboratory exercises is presented during several semesters in courses in a computer engineering undergraduate curriculum in University of Novi Sad and it also applies to Logic Design of Computer Systems II. As one of subjects which are teaching students embedded system design, it was a perfect candidate to join the collaboration between scientific institutions gathered around unified platform on a European level, E2LP [3].

The program of the subject is such that it can easily be adapted for the new platform, with minor changes in the program. Transition to new platform has been recognized as a good moment for structural changes of the course, and instead of only laboratory exercises a small tutorial subcourse was introduced.

Course is following the previous semester course Logic Design of Computer Systems I which is also based on embedded engineering learning platform E2LP [4]]-[6]. It covers the topics of computer architecture and organization and software hardware interfaces. Main emphasis of the lab part is on methods and tools for hardware/software systemon-chip co-design. Laboratory work is project oriented with a set of introductory lab exercises designed to prepare students for project challenges. Students are organized in small project teams (2-4). Project definitions are given in a way to leave a lot of room for various design decisions and implementation methods motivating the students to take the lead.

\section{E2LP UNIFIED BOARD CONCEPT}

E2LP is supporting the following learning objectives:

1. embedded microprocessors \& computer architectures programming (software aspects), 


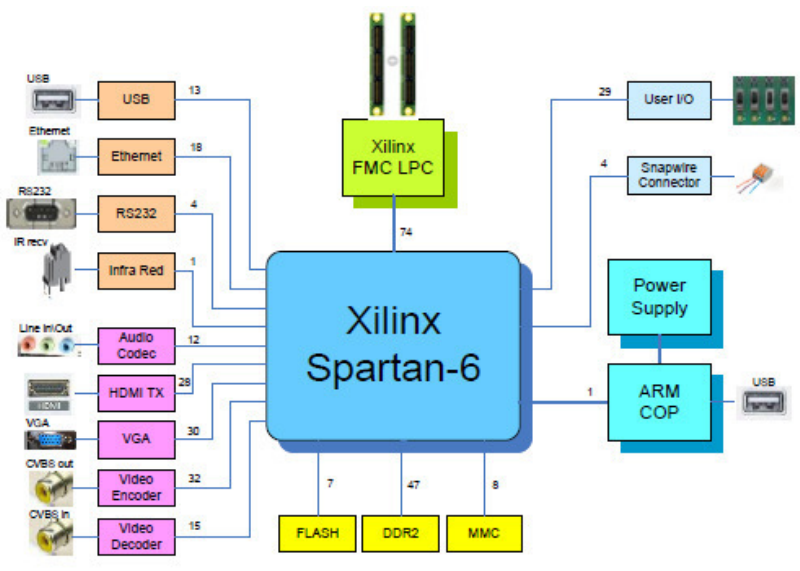

Fig. 1. Architecture of the E2LP base board

2. digital signal processing (audio, video and data) and its real-time implementation,

3. FPGA digital system design and verification,

4. FPGA accelerated computing,

5. networks \& interfaces,

6. system integration.

E2LP platform consists of the Base Board which is presented in Fig. 1, and a set of extension boards which are not relevant for this course.

\section{ARCHITECTURE OF FPGA DESIGN}

Using Xilinx Platform Studio [7] (XPS), the advanced embedded system can be created relatively easy, following the steps from the course documentation. Basic architecture of such system is shown in Fig. 2.

MicroBlaze processor presents a predefined entity in the embedded system. In XPS environment, user can use predefined peripherals available within the XPS, or they can define and implement custom peripheral devices and connect them to the MicroBlaze using XPS tools. Such procedure can be learned very quickly and provides a perfect base for getting introduced into the embedded systems.

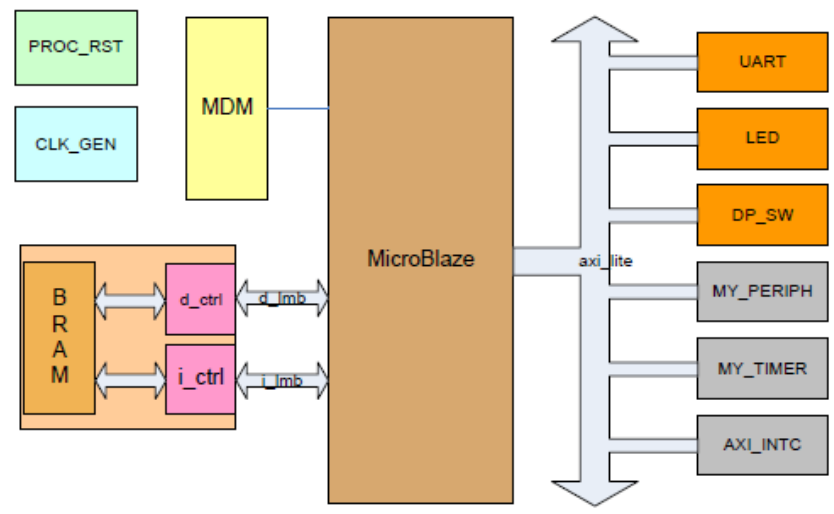

Fig. 2. Simple Xilinx Platform Studio design

\section{Logic Design of Computer Systems II - Course OVERVIEW}

Logic design of computer systems II is aiming to teach students fundamental topics in digital systems:

- Hardware design languages (VHDL)

- Properties of real electronic circuits

- Design of multi-clock systems

- Principles of complex digital system design

Prerequisites for Logic design of computer systems II in general should be basic knowledge of digital electronics and design and VHDL.

Introduction to this course includes basic knowledge of digital electronics and digital design, which is covered with subject Logic Design of Computer Systems 1. In Logic Design of Computer Systems I course, students were introduced to VHDL, Xilinx ISE [7] tool chain, E2LP Board with its application and have used basic user I/O (LED, switches, buttons, LCD, etc.) [4]. In this course a step forward should be made, towards the complex systems.

\section{A. Exercises}

Program of the Logic Design of Computer Systems II is divided into 6 exercises:

1. Clock management techniques, involves command line tool chain tutorial and fundamentals of clock management and distribution, introduction to digital clock manager units and timing constraints. Examples include counters, FSMs and combinational logic

2. VGA interface, includes introduction to VGA interface, text and graphic modes. Students design logic for dynamic display of simple graphical objects and text. Emphasis is on parameterized design.

3. XPS introduction includes introduction to Xilinx XPS (Hardware/Software) tool chain and high level computer system design. Students are instructed to create complex MicroBlaze [8] based computer system with a number of standard XPS peripherals (PS2, UART, memory controllers, etc). Simple C program demonstrates system capabilities.

4. XPS video adapter (hardware part), includes introduction to concepts of memory mapping and bus handling. Students extend existing VGA module functionality and wrap it into an XPS peripheral. Text display is controller from simple MicroBlaze code.

5. XPS video adapter (software part), includes introduction to device driver concept. Students develop a number of low-level driver functions for video adapter peripheral and implement simple console application.

6. XPS advanced exercise, includes introduction to realtime (embedded) programming (interrupts etc) and illustration of system architecture impact on performance (DMA, data cache, bus organization, etc). 
First two exercises are introduction and are not evaluated. Other four exercises are rewarded with 5 points, with 1 additional point for special results (quick or elegant solution of the problem). In total, exercises count as $20 \%$ of the overall grade in this course.

\section{B. Tutorial sub-course}

Tutorial sub-course is organized such that one or a small number of students work on a topic and meet with the instructor weekly for discussion and guidance. For tutorial course we have prepared next tutorials:

1. Factorial coprocessor,

2. Fibonacci coprocessor,

3. VGA stopwatch,

4. Parking machine,

5. Pong game,

6. Snake game,

7. Color VGA,

8. UART VGA terminal,

9. UART LCD terminal.

Depending on the complexity various tutorials were evaluated differently as shown in Table I

Students (23 of them in the Spring 2014 semester), are in position to form teams for each tutorial, with number of team members which is less or equal to number predicted for each tutorial. To have more freedom to be creative and to organize time better, students were allowed to access the laboratory and E2LP platform more often than twice a week, which is defined by the subject program. Weekly meetings with instructor were used for all questions and discussions.

TABLE I.

TUTORIAL COMPLEXITY

MAXIMUM NUMBER OF TEAM MEMBERS AND POINTS

\begin{tabular}{|l|c|c|}
\hline Tutorial name & Team members & Points \\
\hline Factorial coprocessor & 3 & 40 \\
\hline Fibonacci coprocessor & 3 & 40 \\
\hline VGA stopwatch & 3 & 40 \\
\hline Parking machine & 2 & 40 \\
\hline Pong game & 4 & 45 \\
\hline Snake game & 4 & 40 \\
\hline Color VGA & 3 & 45 \\
\hline UART VGA terminal & 3 & 40 \\
\hline UART LCD terminal & 3 & 45 \\
\hline
\end{tabular}

\section{V.TUTORIAL RESULTS}

Here we are presenting some examples of tutorial projects.

\section{A. Stopwatch}

For tutorial named VGA stopwatch (Fig. 3), the goal was to create system that was able to measure elapsed time from the moment when start button is pressed, with precision of $100 \mathrm{~ms}$. The result of measuring should be displayed on VGA screen.

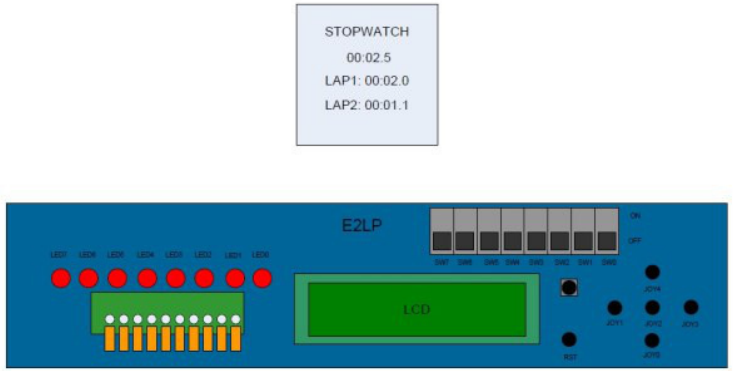

Fig. 3 VGA stopwatch

Besides this, the system should also display overall time and lap time. Lap time is determined with another push button. Third push button is for reset.

Peripheral device, implemented in VHDL, which is connected with MicroBlaze processor with internal AXI bus, is shown in Fig. 4.

This system is measuring time and generating interrupts based on time triggered events and push button states. These interrupts are handled by software part of the system which is generating information and sends it to the VGA screen again using custom made peripheral (Fig. 5).

This solution is successful implementation of all required tasks listed in tutorial documentation. This tutorial was realized by the group of three students, where they did the planning, task scheduling and implementation alone.

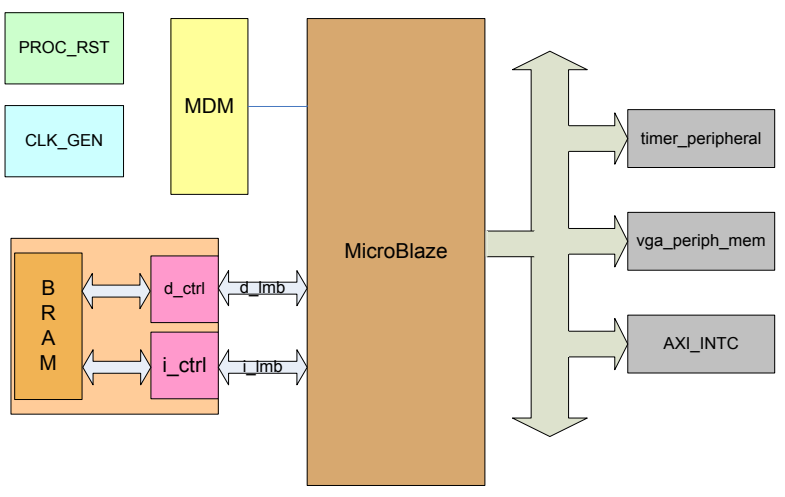

Fig. 4. VGA stopwatch peripheral device

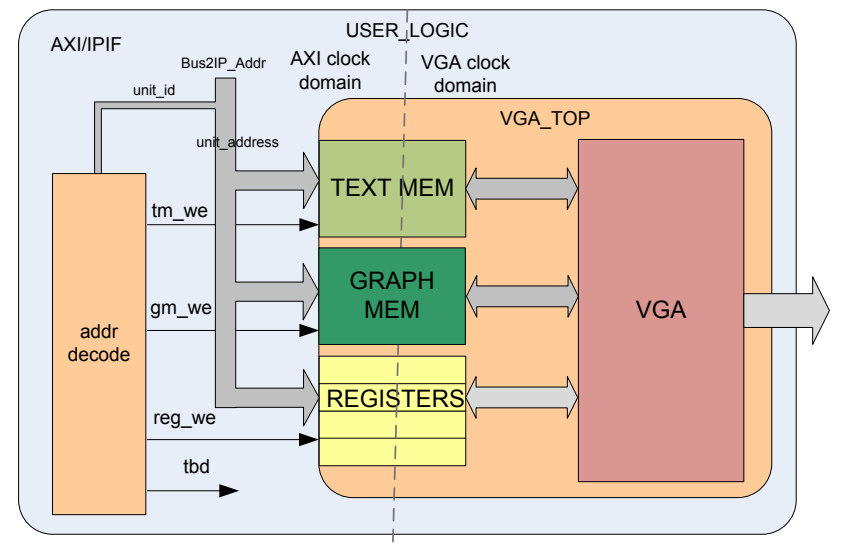

Fig 4 Block diagram of VGA peripheral 


\section{B. Pong Game}

In this tutorial the goal was to create a system that provides playing Pong game for two players. The game is displayed on VGA screen. It should support reception of commands from UART terminal for player 1, command reception from push buttons for player 2, moving of the rackets and ball on the screen and racket-ball collision detection. Block diagram of the system is shown in Fig. 6 and the VGA screen snapshot of the game in Fig. 7.

This tutorial was realized by the group of three students and realization was successful.

\section{STUdENT COMMENTS}

One of the biggest problems was the need to switch from previously used E2LP application for communication with the board. This was necessary because of XPS constant access to the FPGA.

Most of the students commented that introduction to the XPS was too short. They saw this later as a problem during the realization of their tasks. Anyhow, some students expressed that this approach was good enough.

Students found more problems in realization of hardware part of the project and feel more comfortable in software environment. Some tasks were transferred to software part of the system just to avoid problems with hardware part.

Students faced a lot of problems in realization of new peripherals, like UART terminal character reception in software application.

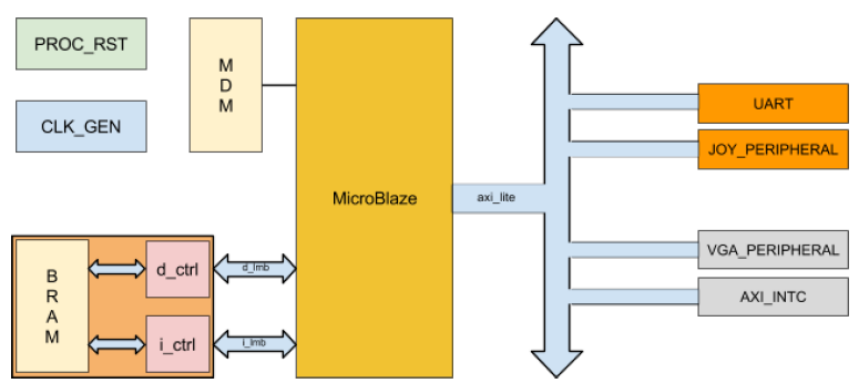

Fig 5 Block diagram of the system architecture

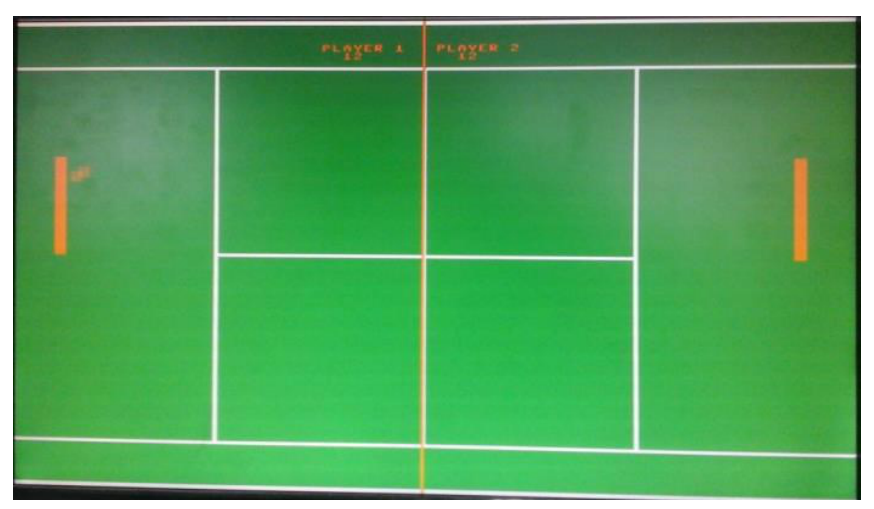

Fig 6 VGA screen snapshot
During the realization of projects which included the use of push buttons, students had problems with bouncing and learned that de-bouncing module is practically an obligatory part of the system.

These are some of many conclusions which are showing what students have learned by only trying to design embedded system by themselves.

\section{CONCLUSION}

In this paper we presented the approach to improvement of students learning of computer system design. It is based on the developed unified platform designed to cover a complete process of embedded systems learning. The presented approach addressed efficiency of active-learning laboratory-based courses.

Academic experience justified the approach. As expected, positive effects were more pronounced in second half of the course where presented approach offered significant improvements without requiring any extra effort from teaching staff. Students were facing problems and tried to solve them alone, which forced them to read documentation more carefully and to even go out of the course documentation, which they saw at the end as a very positive experience. Students had an impression that they learned a lot, and they felt more confident after successful realization of the tasks.

As part of future work, we plan to introduce new tutorials in the course that will integrate all student knowledge from embedded system design. Another direction of the future work is to prepare a re-design of the teaching platform in order to respond to students' comments.

\section{REFERENCES}

[1] Holbert, K.E., Karady, G.G., "Strategies, Challenges and Prospects for Active Learning in the Computer-Based Classroom", IEEE Transactions on Education, Volume 52, Issue 1, Feb. 2009 pp. 31 38

[2] Ron Schneiderman, "Economy and Shortages Affect the European Job Outlook", IEEE Spectrum, March 2010, Available from (accessed on 2011-12-10) http://spectrum.iee.org/at-work/techcareers/economy-andshortages-affect-the-european-job-outlook/0

[3] Temerinac, M.; Kastelan, I; Skala, K.; Medved Rogina, B.; Reindl, L.; Souvestre, F.; Anastassova, M.; Szewczyk, R.; Piwinski, J.; Benito, J.R.L.; Artetxe Gonzalez, E.; Teslic, N.; Sruk, V.; Barak, M., "E2LP: A Unified Embedded Engineering Learning Platform," Digital System Design (DSD), 2013 Euromicro Conference on , vol., no., pp.266,271, 4-6 Sept. 2013

[4] Kastelan, I; Katona, M.; Majstorovic, D.; Neborovski, E., "Students perspective of the embedded engineering learning platform - A case study in digital design," MIPRO, 2011 Proceedings of the 34th International Convention, vol., no., pp.1178,1182, 23-27 May 2011

[5] Majstorovic, D.; Neborovski, E.; Katona, M., "A cross-curriculum embedded engineering learning platform," MIPRO, 2010 Proceedings of the 33rd International Convention, vol., no., pp.1039,1044, 24-28 May 2010

[6] Kastelan, I; Majstorovic, D.; Nikolic, M.; Eremic, J.; Katona, M., "Laboratory exercises for embedded engineering learning platform," MIPRO, 2012 Proceedings of the 35th International Convention , vol., no., pp.1113,1117, 21-25 May 2012

[7] Xilinx design tools, http://www.xilinx.com/products/design-tools/

[8] Xilinx MicroBlaze, http://www.xilinx.com/tools/microblaze.htm 